\section{EL CÁNCER GINECOLÓGICO}

\author{
Antonio González-Martín \\ MD Anderson Cancer Center \\ agonzalezm@seom.org
}

\section{GYNAECOLOGICAL CANCER}

Cómo citar este artículo/Citation: González-Martín, A. (2015). “El cáncer ginecológico". Arbor, 191 (773): a237. doi: http:// dx.doi.org/10.3989/arbor.2015.773n3007
Copyright: (C) 2015 CSIC. Este es un artículo de acceso abierto distribuido bajo los términos de la licencia Creative Commons Attribution-Non Commercial (by-nc) Spain 3.0.

Recibido: 9 diciembre 2014. Aceptado: 24 abril 2015.

RESUMEN: El cáncer ginecológico es un problema de salud en Europa de primer orden en la medida en que en su conjunto, e incluyendo específicamente el cáncer de ovario, de útero y de cuello uterino, representa la tercera causa de cáncer en la mujer en Europa, por detrás del cáncer de mama y el cáncer colorrectal, y la tercera causa de muerte por cáncer tras el cáncer de mama y cáncer de pulmón. La mejora en estas cifras pasa por la implementación de las medidas preventivas disponibles, especialmente en cáncer de cuello uterino, el diagnóstico precoz cuando es posible, la aplicación de la mejor terapéutica por equipos multidisciplinares especializados, y el conocimiento de las alteraciones moleculares de estas enfermedades, como base para el desarrollo de terapias personalizadas y eficientes.

PALABRAS CLAVE: cáncer de cuello uterino; cáncer de ovario; cáncer de útero.
ABSTRACT: Gynecological cancer is a major health problem in Europe, as taken together, ovarian cancer, cancer of the uterus and cervical cancer, represent the third cause of cancer in women in Europe, after breast cancer and colorectal cancer, and also the third cause of death from cancer after breast cancer and lung cancer. Improvement in these data entails the implementation of the prophylactic measures available. Especially in the case of cervical cancer, early diagnosis whenever it is possible, the administration of the best treatment by specialised multidisciplinary teams and knowledge of the molecular alterations associated with these diseases are required as a base for the development of personalised and effective treatments. 


\section{CÁNCER DE CUELLO UTERINO (CÉRVIX)}

El cáncer de cérvix o cuello de útero es una enfermedad única en el contexto de la oncología porque tiene unos factores de riesgo muy bien establecidos, posee un largo periodo pre-invasivo durante el cual puede ser detectado por técnicas de cribado, se conoce muy bien el agente etiológico y existen vacunas preventivas disponibles.

\subsection{Datos epidemiológicos}

El cáncer de cérvix o cuello uterino es un problema médico de orden mundial en la medida en que representa la tercera causa de cáncer en la mujer en todo el mundo con unos 500.000 casos nuevos al año y causa unas 250.000 muertes anuales, lo que le convierte en la 4a causa de muerte por cáncer en la mujer en todo el mundo. La mayoría de los casos nuevos y muertes por cáncer de cérvix se continúan produciendo en países en vías de desarrollo debido a la ausencia de campañas de cribado efectivas y la escasa o nula implementación de la vacunación preventiva. Los países subdesarrollados o en vías de desarrollo del África sub-Sahariana, América Latina y Caribe, y regiones centrales y del sureste Asiático son los que registran el mayor número de casos, y en muchos de estos países es la principal causa de muerte por cáncer en la mujer. Por el contrario, en los países desarrollados se ha producido un descenso de mortalidad de un $70 \%$ en los últimos 30-50 años por cáncer de cérvix debido a la implementación de técnicas de cribado.

La edad media de presentación está en torno a los 50 años y solo el $10 \%$ de los casos ocurren en pacientes de más de 75 años. De hecho, en la franja de edad de los 15 a los 44 años es la segunda causa de cáncer en la mujer por detrás del cáncer de mama. El tipo histológico más frecuente es el carcinoma epidermoide o escamoso, sin embargo en los países industrializados está aumentando la incidencia de adenocarcinoma que puede alcanzar hasta el $25 \%$ de los casos en algunos países.

\subsection{El virus del papiloma humano (HPV) como agen- te etiológico del cáncer de cérvix}

El virus del papiloma humano (HPV) es la causa fundamental del carcinoma de cérvix, tanto del tipo epidermoide como del adenocarcinoma. Además se ha relacionado con el cáncer vulvar, vaginal, anal, cáncer de pene y cánceres de orofaringe, cavidad oral y quizás laringe. El HPV es un virus DNA que se transmite por vía sexual e induce lesiones proliferativas en el epitelio cutáneo y mucoso. Pertenece a la familia Papovaviridae y se han identificado más de 200 subtipos de los cuales 18 son oncogénicos. Los tipos de HPV oncogénicos más comunes en pacientes con carcinoma de cérvix son los tipos $16,18,31,33,35,39,45$, $51,52,56,58,59,68,73$, y 82 . En conjunto los tipos 16 y 18 son los más frecuentes y en concreto responsables del $90 \%$ de los carcinomas de cérvix y del $50 \%$ $60 \%$ de las lesiones pre-invasivas CIN grado 2 y 3.

La prevalencia de infección cervical por HPV de alto riesgo entre las mujeres jóvenes (en torno a los 25 años) es del 20 al $60 \%$ dependiendo del área geográfica. Esta incidencia desciende con la edad en la medida en la que el sistema inmune logra vencer la infección. De hecho, la mayoría de mujeres (90\%) con infección por un tipo de HPV no tienen evidencia de la persistencia de dicho tipo a los 12-24 meses. Además, la reinfección por el mismo tipo es muy infrecuente. Por todo ello, solo una minoría de lesiones cervicales con infección por HPV progresará a carcinoma.

La infección persistente del HPV ocurre por el acantonamiento del virus en la zona de transformación cervical donde el epitelio columnar da paso al epitelio estratificado del cuello uterino. La proliferación incontrolada de las células epiteliales observada en las lesiones cervicales provocadas por HPV ha sido atribuido a los oncogenes virales E6 y E7. El mecanismo por el cual estos genes de HPV estimulan la progresión del ciclo celular está relacionado con la inactivación de la proteína del retinoblastoma (pRb) y de p53. La acción de E6 induce la inactivación de p53, alterando la respuesta normal al daño del DNA y favoreciendo la acumulación de mutaciones, a la vez que E7 mantiene el estímulo mitótico celular y con ello permite la replicación del DNA viral.

\subsection{Factores de riesgo}

Las pacientes que mantienen una infección persistente por un tipo de HPV de alto riesgo son las que desarrollarán carcinoma de cérvix. Típicamente el tiempo que transcurre desde que se produce la infección hasta el desarrollo de una lesión premaligna (CIN 3) y finalmente un carcinoma invasor es de 15 a 20 años. Se han descrito varios factores de riesgo y factores coadyuvantes que pueden actuar en tan largo intervalo de tiempo favoreciendo el desarrollo de carcinoma invasivo.

Al tratarse de una infección de transmisión sexual hay múltiples factores de riesgo que se han asociado a la probabilidad de adquirir la infección por VPH. De 
ellos destaca un número elevado de parejas, primera relación antes de los 18 años, primer parto antes de los 18 años, tener 4 o más partos, el uso de anticonceptivos orales durante más de 5 años y la presencia de otras infecciones de transmisión sexual, como por ejemplo la Clamydia o el VIH (virus inmunodeficiencia humana). De hecho la inmunodeficiencia causada por VIH es un factor de riesgo muy relevante, y además el desarrollo de cáncer de cérvix es un criterio diagnóstico de SIDA en una portadora de infección por VIH. Además de estos factores, el abuso del tabaco se ha asociado a un aumento del riesgo que a su vez se incrementa en función del número de cigarrillos que se consumen. En contraposición a todo lo anterior, el uso del preservativo puede ayudar a reducir en un $70 \%$ el riesgo de transmisión.

\subsection{Prevención de cáncer de cuello uterino: situación actual de la vacunación y del cribado poblacional}

\subsubsection{Vacunación}

En este momento se dispone de dos vacunas aprobadas para la prevención de infección por VPH: Gardasil que previene frente a los serotipos de VPH 16, 18,6 y 11 y Cervarix que previene frente a serotipos 16 y 18 . Ambas vacunas previenen las lesiones preneoplásicas y el carcinoma de cérvix causados por VPH 16 y 18, adicionalmente Gardasil previene las verrugas genitales causadas por VPH 6 y 11, y Cervarix parece tener capacidad de prevención cruzada con otros serotipos oncogénicos. Su empleo se recomienda en niñas entre 9 y 14 años para obtener el máximo efecto protector antes de que se produzca la primera relación sexual en un periodo de la vida, de los 10 a los 15 años, en el que la respuesta inmune es muy elevada. Aunque no existe un consenso internacional absoluto, también se recomienda la vacunación antes de los 26 años independientemente de la actividad sexual, si bien este criterio no se ha incluido en los calendarios vacunales poblacionales (McGraw y Ferrante, 2014).

Las tasa de implementación de la vacunación y su inclusión en el calendario vacunal varía de unos países a otros, pero se ha estimado que es preciso vacunar al $70-80 \%$ de las niñas en edad pre-puberal para obtener inmunidad de grupo o poblacional. Incluso, algunos autores han preconizado la vacunación de los varones para obtener este objetivo dado que de alguna manera actúan como reservorio del VPH. Sin embargo hasta este momento no se puede considerar esta maniobra como coste-efectiva.

\subsubsection{Cribado poblacional}

El empleo de la citología vaginal o test de Papanicolau como método de cribado poblacional ha contribuido a una reducción drástica de la mortalidad por cáncer de cuello de útero en los países industrializados. Una de las limitaciones de este test es la elevada tasa de falsos positivos. Sin embargo, el empleo de técnicas de detección del VPH en mujeres de más de 30 años tiene una elevada sensibilidad y su empleo junto con la citología permite la optimización del cribado poblacional. El advenimiento del test de detección de VPH ha modificado las recomendaciones de los programas de cribado que originariamente incluía la realización de una citología anual. La mayoría de sociedades ha desarrollado sus propias guías de cribado. En el año 2012 la American Cancer Society (ACS), American Society for Colposcopy and Cervical Pathology (ASCCP), y la American Society of Clinical Pathology (ASCP), junto con the United States Preventive Services Task Force (USPSTF) desarrollaron una guía de consenso para población general cuyas principales recomendaciones fueron:

1) comenzar el cribado a los 21 años,

2) de los 21 a los 29 años realizar citología cada 3 años,

3) de los 30 a los 65 años realizar co-test con citología y determinación de VPH cada 5 años, en caso de que no se disponga de posibilidad de realizar test de VPH se considera adecuada la realización de citología cada 3 años,

4) interrumpir el cribado a los 65 años por el bajísimo riesgo de desarrollar carcinoma de cuello uterino en las mujeres que adquieran nueva infección por VPH a partir de esta edad.

Estas recomendaciones también se mantienen aún para las mujeres que hayan sido vacunadas, y no se espera que cambien hasta que hayan pasado por lo menos 10 años desde la implementación de la vacunación (McGraw y Ferrante, 2014).

Dado que este tipo de cribado poblacional no está al alcance de los países no desarrollados, se han desarrollado métodos alternativos que están ganando popularidad por su sencillez y sus resultados. En concreto el más estudiado es el test de inspección visual con ácido acético, que puede ser realizado por personal no sanitario con el adecuado entrenamiento y cuya clara utilidad fue demostrada en un gran estudio poblacional llevado a cabo en la India. 


\subsection{Tratamiento del cáncer de cuello uterino: avan- ces recientes en terapia loco-regional}

El tratamiento del cáncer de cuello uterino depende esencialmente del estadio en que se encuentra la enfermedad. En la tabla 1 se resume el tratamiento considerado estándar y las posibles alternativas más novedosas en los distintos estadios de la enfermedad.

\subsubsection{Avances en tratamiento loco-regional}

Uno de los avances en el tratamiento quirúrgico del cáncer de cérvix ha consistido en la incorporación de cirugía preservadora de la fertilidad para aquellas pacientes jóvenes con tumores localizados. La técnica se denomina traquelectomía y consiste en la extirpación del cérvix y el parametrio manteniendo el fondo uterino que se reconecta a la vagina. Las pacientes candidatas deben tener tumores en estadios precoces (IA2 o IB1), menores de $2,5 \mathrm{~cm}$ y ausencia de afectación ganglionar. Con esta técnica en pacientes seleccionadas se consiguen los mismos resultados oncológicos que con una histerectomía, y un $80 \%$ de las pacientes consigue quedar embarazada. De estas, aproximadamente el $75 \%$ de los embriones consiguen llegar al tercer trimestre, y aquellos que se pierden lo hacen fundamentalmente en el primer trimestre (20\%). En este momento se está desarrollando un ensayo clínico prospectivo en MD Anderson denominado ConCerv con el fin de validar este concepto.

Con respecto a los tumores de cérvix de más de $4 \mathrm{~cm}$ o en tumores con afectación parametrial, tercio inferior de vagina, pared pélvica o mucosa rectal o vesical (estadios IIB-IVA), el tratamiento estándar consiste en la administración concurrente de radioterapia y quimioterapia basada en cisplatino. La dosis habitual de cisplatino es de $40 \mathrm{mg} / \mathrm{m} 2$ una vez por semana mientras dura la radioterapia. Un meta-análisis realizado por la
Cochrane sobre todos los ensayos aleatorizados que habían comparado la quimio-radioterapia concurrente con la radioterapia para el carcinoma de cérvix avanzado identificó 21 estudios publicados que incluyeron 4921 pacientes. La quimio-radioterapia proporcionó un beneficio absoluto en supervivencia libre de progresión y supervivencia global del $13 \%$ y $10 \%$ respectivamente.

Aún se encuentra en fase de estudio el papel de la quimioterapia neoadyuvante, es decir previa a la quimio-radioterapia o a la cirugía, así como la quimioterapia adyuvante, es decir después de la quimioradioterapia en aquellos casos con factores de alto riesgo de recaída. Acaba de completarse un estudio conducido por la European Organization for Research and Treatment of Cancer (EORTC) que exploraba el concepto de la neoadyuvancia y aún sigue en marcha un estudio realizado en el seno del Gynecologic Cancer InterGroup (GCIG) liderado por el grupo australiano ANZOG para testar el papel de la adyuvancia. Se espera que los resultados de estos estudios nos aclaren el papel de la quimioterapia en ambos contextos.

\subsection{Avances en el tratamiento del cáncer de cuello uterino recidivado o metastásico}

Aunque en nuestro medio la mayoría de las pacientes con carcinoma de cérvix se diagnostican en estadios precoces que permiten la aplicación de tratamientos loco-regionales curativos, más de un tercio de las pacientes con enfermedad localmente avanzada fallecen por la presencia de metástasis desde el comienzo o el desarrollo de una recurrencia tras el tratamiento local. Salvo aquellas pacientes que presentan una recurrencia pélvica central que pueda ser rescatada con cirugía de exanteración o radioterapia, la mayoría de las pacientes con enfermedad metastásica o recurrente deben ser valoradas para un tratamiento paliativo con quimioterapia.

Tabla 1. Tratamiento del cáncer de cérvix de acuerdo al estadio

\begin{tabular}{|c|c|c|c|c|}
\hline Estadio & IA1 & IA2 & IB1, IIA & IIB-IVA \\
\hline Tratamiento estándar & $\begin{array}{l}\text { Conización o histerec- } \\
\text { tomía simple +/- sal- } \\
\text { pingo-ooforectomía y } \\
\text { PLND en caso de ILV }\end{array}$ & $\begin{array}{l}\text { Conización / traquelec- } \\
\text { tomía o histerectomía } \\
\text { radical modificada y } \\
\text { PLND }\end{array}$ & $\begin{array}{l}\text { Histerectomía radical } \\
\text { modificada y PLND } \\
\text { (traquelectomía en ca- } \\
\text { sos seleccionados) }\end{array}$ & $\begin{array}{l}\text { Quimio-radiote- } \\
\text { rapia basada en } \\
\text { cisplatino }\end{array}$ \\
\hline Tratamiento a considerar & $\begin{array}{l}\text { Cirugía conservadora } \\
\text { de fertilidad (traque- } \\
\text { lectomía) }\end{array}$ & $\begin{array}{l}\text { Quimio-radioterapia } \\
\text { adyuvante en caso de: } \\
\text { ILV, grado 3, ganglios } \\
\text { afectados o márgenes } \\
\text { de resección afectados }\end{array}$ & $\begin{array}{l}\text { Quimio-radioterapia } \\
\text { adyuvante en caso de: } \\
\text { ILV, grado 3, ganglios } \\
\text { afectados o márgenes } \\
\text { de resección afectados }\end{array}$ & $\begin{array}{l}\text { QT neoadyuvante } \\
\text { seguido de QT-RT }\end{array}$ \\
\hline
\end{tabular}

ILV: invasión linfovascular; PLND: linfadenectomía pélvica; QT: quimioterapia; QT-RT: quimio-radioterapia concurrente. 
A lo largo de los últimos 15 años se han llevado a cabo varios ensayos clínicos que posicionaron la combinación de paclitaxel y cisplatino como el tratamiento estándar de las pacientes con cáncer de cérvix metastático o recurrente. Sin embargo los resultados del tratamiento con dicho esquema en este contexto de la enfermedad distan mucho de ser satisfactorios pues se alcanza una mediana de supervivencia que no suele superar los 12 meses. Cifra que es aún mucho menor en pacientes con criterios de alto riesgo definidos por la existencia de síntomas, presencia de recaída pélvica, haber recibido un tratamiento de quimioradioterapia pélvica o presentar una recaída antes de un mes. Por todo lo anteriormente citado, el cáncer de cérvix metastático o recurrente es una enfermedad no curable y su tratamiento es una necesidad médica no cubierta.

La mayor innovación reciente en este campo ha sido la incorporación de la terapia antiangiogénica a la quimioterapia convencional (Tewari y Monk, 2014). La neoangiogénesis, o desarrollo de nuevos vasos sanguíneos, juega un papel esencial en la progresión del cáncer de cérvix. Tanto las proteínas E6 como E7 por diferentes mecanismos producen un incremento en la producción del factor de crecimiento vasculo-endotelial (VEGF) que es un factor esencial en la promoción y mantenimiento de la neovasculatura que requiere el tumor para desarrollarse. Afortunadamente empezamos a disponer de medicamentos capaces de bloquear el VEGF como bevacizumab que es un anticuerpo monoclonal humanizado, o medicamentos con actividad inhibidora de tirosina kinasa capaces de bloquear la activación del receptor de VEGF. En un estudio recientemente publicado, en el que participó el Grupo Español de Investigación en Cáncer de Ovario (GEICO), se demostró que la asociación de bevacizumab al tratamiento de quimioterapia estándar con paclitaxel y cisplatino produce un incremento significativo de la supervivencia global de las pacientes, alcanzando una mediana de supervivencia que supera los 17 meses (Tewari et al., 2014). Esta es la primera vez que un ensayo clínico con terapia biológica muestra un incremento en la supervivencia global de una enfermedad tan devastadora como el carcinoma de cérvix en situación metastásica o recurrente.

Finalmente, otras vías de desarrollo clínico de terapia biológica actualmente en marcha en cáncer de cérvix metastático incluyen: terapia antiangiogénica con inhibidores de tirosina kinasa, inhibidores de la vía de Notch, inhibidores de mTOR, inhibidores de PARP y la inmunoterapia.

\section{CÁNCER DE ENDOMETRIO}

\subsection{Una visión general del cáncer de endometrio}

El cáncer de endometrio es el tumor ginecológico más frecuente y el cuarto cáncer más frecuente después del cáncer de mama, pulmón y colorrectal en las mujeres de EEUU y Europa. La mayoría de pacientes con cáncer de endometrio se diagnostican en etapas precoces cuando la enfermedad está aún confinada al cuerpo uterino, lo que hace que las tasas de curación sean muy elevadas. El hecho de que el síntoma inicial de la enfermedad sea un sangrado vaginal inapropiado, que generalmente motiva la visita al ginecólogo, es sin duda uno de los factores que contribuye a la alta tasa de diagnósticos precoces de la enfermedad.

El tipo histológico más frecuente es el adenocarcinoma endometriode y los principales factores pronóstico de esta enfermedad incluyen la edad, la raza, el estadio, el grado, la ploidía, la profundidad de la invasión, el tamaño del tumor, la expresión de receptores hormonales y el subtipo histológico. A pesar de que el pronóstico de esta enfermedad es globalmente favorable, algunas pacientes pueden tener un comportamiento muy agresivo, especialmente aquellas con tumores de alto grado, invasión profunda del miometrio o subtipos histológicos más agresivos como el seroso papilar o de células claras.

El cáncer de endometrio ocurre con más frecuencia en mujeres post-menopáusicas, y el factor de riesgo más importante es la exposición prolongada a estrógenos. De ahí que la obesidad, que genera un incremento de la producción de estrógenos en los adipocitos, sea un claro factor de riesgo identificado. Otro factor etiológico identificado es la toma de tamoxifeno, si bien la incidencia es tan baja como el 1.6/1000 mujeres que toman este fármaco.

Aunque la mayoría de los cánceres de endometrio son esporádicos, algunas mujeres tienen una predisposición genética a esta enfermedad. El síndrome más frecuentemente asociado al cáncer de endometrio es el síndrome hereditario de cáncer colorrectal no-polipósico también conocido como síndrome de Lynch que es un síndrome de susceptibilidad autosómica dominante que se produce por una mutación de uno de los genes de reparación de DNA, en concreto en MLH1, MSH2, MSH6 o PMS2. El riesgo de desarrollar cáncer de endometrio a lo largo de la vida en portadoras del síndrome de Lynch es del $40-60 \%$ y suele aparecer en mujeres jóvenes. 
EL cáncer de endometrio se ha dividido en dos grandes grupos. El tipo I, que es el más frecuente e incluye el adenocarcinoma endometriode que típicamente surge de una hiperplasia endometrial y es estrógeno dependiente, del que hablaremos en esta sección. El tipo II, es menos frecuente e incluye variantes más agresivas como el carcinoma seroso papilar, el carcinoma de células claras y el carcino-sarcoma.

\subsection{Controversias en el tratamiento del cáncer de endometrio}

\subsubsection{La importancia de la estadificación ganglionar}

El tratamiento esencial del cáncer de útero es la histerectomía con doble anexectomía. Adicionalmente, la afectación de ganglios linfáticos regionales es el factor pronóstico más importante en cáncer de endometrio, y además influye en el tratamiento complementario tras la cirugía. Se han identificado varios factores clinicopatológicos que incrementan el riesgo de afectación ganglionar, entre ellos destacan el alto grado (aumenta en 6 veces el riesgo), la invasión miometrial profunda (aumenta en 5 veces el riesgo), la afectación del espacio linfovascular, y la invasión del estroma cervical.

La manera de estadificar el cáncer de endometrio es quirúrgica, y se dispone de evidencia suficiente para afirmar que la laparoscopia se puede considerar un procedimiento estándar frente a la cirugía abierta. Sin embargo la extensión del procedimiento para conocer la afectación ganglionar continua siendo un asunto controvertido. Para algunos ginecólogos oncólogos es suficiente con hacer un muestreo linfático en pacientes de alto riesgo (aquellas portadoras de tumores con invasión profunda o de alto grado). Por otro lado, para otros se debe realizar una linfadenectomía ganglionar pélvica y para-aórtica de rutina pues hasta el 18\% de pacientes con tumores grado 1 puede tener afectación ganglionar oculta. Sin embargo, los dos estudios aleatorizados que se han realizado hasta la fecha en los que se ha explorado el papel de la linfadenectomía de rutina no han demostrado que tengan ningún impacto en la supervivencia de las pacientes. No obstante, algunas críticas metodológicas hacen que persista la controversia. Una vía de desarrollo y posible resolución en este conflicto es la realización de la técnica de ganglio centinela. Ello permitiría seleccionar a las pacientes que deben de recibir la linfadenectomía, evitando las complicaciones inherentes al procedimiento a aquellas pacientes que no tengan afectación ganglionar. Sin embargo, este es un procedimiento aún experimental (Burke et al., 2014b; Wright et al., 2012).

\subsection{2. ¿Tiene algún papel la cirugía en el tratamiento de las etapas avanzadas (estadios III y IV)?}

La visión del papel de la cirugía en las etapas avanzadas (estadios III y IV) ha cambiado en los últimos años. Estas pacientes se han tratado tradicionalmente de forma convencional con quimioterapia o radioterapia. Sin embargo, hoy disponemos de estudios que muestran que aquellas pacientes con tumores en etapas III y IV que son sometidas a una cirugía citorreductora en la que no quede enfermedad residual alcanzan una mediana de supervivencia que puede ser el doble que en las pacientes en las que queda enfermedad residual (40 meses vs 19 meses). Aunque esta evidencia proviene de estudios retrospectivos y esta aproximación no ha sido validada de forma prospectiva, debe ser tenida en cuenta a la hora de abordar el manejo terapéutico de pacientes con estadios III y IV en los comités multidisciplinares de tumores ginecológicos (Shih et al., 2011).

\subsubsection{Papel de la radioterapia adyuvante}

Si hay un área de controversia persistente en el campo de la oncología ginecológica, este es el papel que a día de hoy tiene la radioterapia adyuvante tras el tratamiento quirúrgico. $Y$ aún se complica más si tenemos en cuenta que nos enfrentamos a dos tipos de pacientes: las que han recibido una estadificación ganglionar completa y los que no.

Se ha aceptado de forma general que las pacientes con tumores endometrioides grado 1-2 y con invasión de menos de la mitad del miometrio (estadio IA) tiene muy bajo riesgo de recaída $(<5 \%)$ independientemente del tipo de estadificación que se haga, y que por tanto no requieren tratamiento adyuvante. Sin embargo, para el resto de pacientes existe aún controversia sobre el impacto de la terapia adyuvante tras una cirugía de estadificación adecuada y la selección de pacientes para dicha terapia.

Los principales factores asociados al riesgo de recaída son la edad, el grado tumoral, la profundidad de la invasión miometrial y la presencia de invasión linfovascular. La radioterapia adyuvante en pacientes con estos factores de riesgo ha mostrado que puede reducir las recaídas loco-regionales en un $64 \%$ pero no impacta en la supervivencia global ni en la supervivencia relacionada con la enfermedad. Esto se ha explicado, porque las pacientes con recaída local pueden ser rescatadas y curadas con radioterapia, y de hecho tienen mejor pronóstico que las recaídas locales en pacientes que han recibido previamente irra- 
diación. En la tabla 2 se recogen los resultados de los principales estudios que han explorado el papel de la radioterapia adyuvante en pacientes con carcinoma de endometrio de riesgo intermedio y alto. Por su parte la braquiterapia ha demostrado que puede alcanzar la misma tasa de control local que la radioterapia externa pélvica, con un periodo libre de progresión y supervivencia global que estimamos razonable, pero con una menor tasa de eventos adversos y una mejor calidad de vida (Burke et al., 2014b; Susumu et al., 2008).

\subsection{4. ¿Tiene algún papel la quimioterapia adyuvante?}

Un subgrupo de pacientes con estadios I y II tienen alto riesgo de recaída a distancia, especialmente aquellas que presentan alto grado, invasión de más del $50 \%$ de miometrio o invasión linfovascular. En estas pacientes se ha postulado la quimioterapia adyuvante como una estrategia para reducir el riesgo de recaída a distancia. Sin embargo, no se dispone de suficiente evidencia aún como para realizar recomendaciones firmes para incluirlo como tratamiento estándar.

En pacientes con estadios avanzados (etapas FIGO III-IV) que se habían sometido a cirugía y en las que no quedó enfermedad residual mayor de $2 \mathrm{~cm}$, un tratamiento de quimioterapia con adriamicina y cisplatino resultó superior a un tratamiento con radioterapia abdominal total en términos de supervivencia global con un incremento de la tasa de supervivencia a 5 años del 13\% (42\% vs 55\% a favor de quimioterapia), si bien el esquema de quimioterapia indujo una mayor toxicidad.

Un estudio Japonés aleatorizó pacientes con estadios IC a IIIC a tratamiento con radioterapia o quimioterapia adyuvante con cisplatino-adriamicinaciclofosfamida. Aunque la supervivencia fue similar globalmente, se identificó un subgrupo de pacientes con incremento en supervivencia global (estadio IC y > 70 años o grado 3, y estadio II o pacientes con citología positiva con invasión de $>50 \%$ de miometrio). Finalmente, un estudio de la European Organisation for Research and Treatment of Cancer (EORTC) y Nordic Society of Gynecological Oncology (NSGO) comparó la asociación de radioterapia adyuvante con quimioterapia (varios regímenes) frente a radioterapia sola en pacientes con cáncer de endometrio estadio I-IIIC, observando un beneficio a favor de la quimioterapia con una reducción del riesgo de recaída del 36\% [Hazard ratio de 0.64 (95\% Cl 0.41-0.99)]. Sin embargo, el $27 \%$ de las pacientes en la rama de quimioterapia no pudieron completar el tratamiento (Hogberg et al., 2010).
Actualmente hay varios estudios en marcha que exploran e intentan aclarar el papel de la adición de quimioterapia adyuvante en el carcinoma de endometrio en etapas precoces. El estudio GOG-249 compara la radioterapia pélvica completa con la combinación de braquiterapia y paclitaxel-carboplatino, y el estudio PORTEC-3 compara radioterapia pélvica con radioterapia pélvica más quimioterapia.

\subsection{Avances en el tratamiento médico del cáncer de endometrio metastásico o recurrente}

Para las pacientes con recaída de cáncer de endometrio que no son subsidiarias de un tratamiento de rescate quirúrgico o con radioterapia, y en aquellas pacientes con enfermedad metastásica, el tratamiento de elección es un tratamiento sistémico paliativo (Burke et al., 2014a; Burke et al., 2014b; Wright et al., 2012).

En pacientes con tumores de bajo grado histológico (grado 1) y presencia de expresión de receptores de progesterona el uso de progestágenos a dosis bajas puede alcanzar una tasas de respuesta del $15-30 \%$ y es un tratamiento especialmente indicado en pacientes ancianas o con comorbilidades. En el resto de las pacientes la quimioterapia es el tratamiento de elección. Hoy día disponemos de varios regímenes con distinta actividad y grado de toxicidad. La adriamicina en monoterapia fue superada por la combinación de adriamicina y ciplatino en términos de tasa de respuesta pero no en supervivencia global. Posteriormente, un estudio desarrollado por el grupo GOG demostró que la combinación de paclitaxel-cisplatino-adriamicina (TAP) era superior a cisplatino-adriamicina en términos de tasa de respuesta ( $34 \%$ vs $57 \%$ ) y en términos de supervivencia global ( 12.3 meses vs 15.3 meses) a costa de una toxicidad substancialmente mayor. Por este motivo este esquema no se ha estandarizado como el régimen habitual. En cambio, la combinación de paclitaxel y carboplatino (TC) que induce una tasa de respuesta en torno al $40 \%$ en estudios fase II se ha convertido en el régimen más usado por su mejor tolerabilidad y conveniencia de uso. Un estudio del GOG cuyos resultados están pendientes ha comparado ambos esquemas de tratamiento (TAP vs TC).

Finalmente, en la actualidad se están desarrollando varios ensayos clínicos con nuevos fármacos, especialmente con inhibidores de la vía de señalización intracelular PI3K-mTOR-AKT debido a que aproximadamente el $50 \%$ de las pacientes tiene una alteración en esta vía, y con terapia antiangiogénica. Sin embargo, aunque se ha observado cierta actividad de estos fármacos en estudios precoces, hasta el momento no se dispone de datos de estudios aleatorizados que hayan permitido su incorporación a la práctica asistencial. 


\begin{tabular}{|c|c|c|c|c|c|c|}
\hline 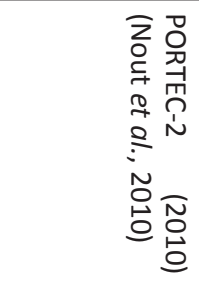 & 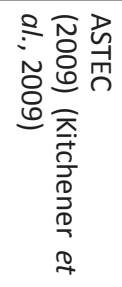 & 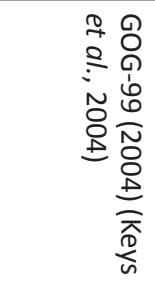 & 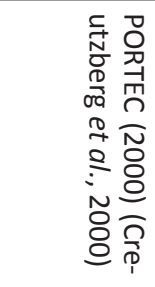 & 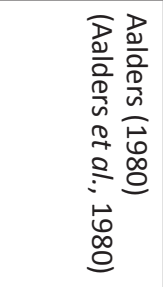 & & $\begin{array}{l}\text { 罢 } \\
\text { 产. } \\
\text { o. }\end{array}$ \\
\hline 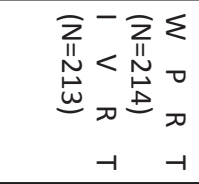 & 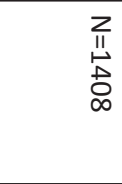 & 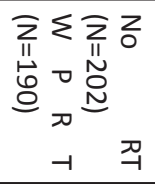 & 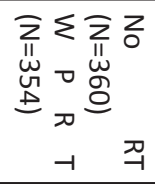 & 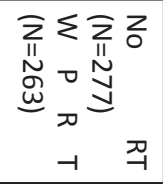 & & $z$ \\
\hline 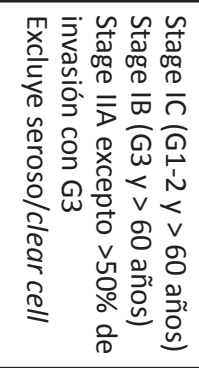 & 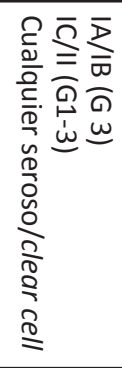 & 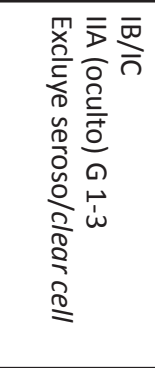 & 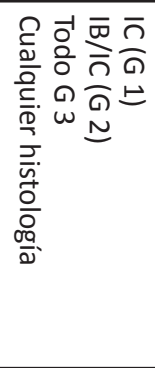 & 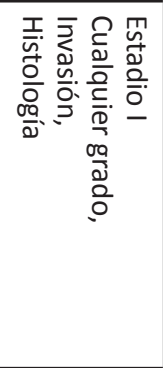 & & 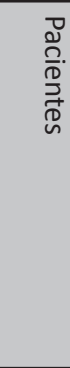 \\
\hline z & z & $\simeq$ & z & z & & 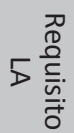 \\
\hline 1 & 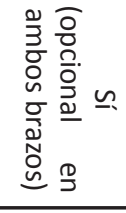 & zo & zo & 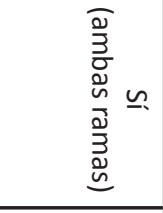 & & 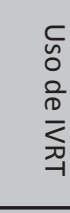 \\
\hline 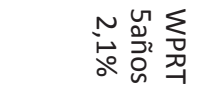 & 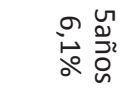 & ○ & 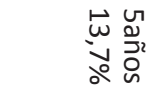 & 1 & $\begin{array}{l}z \\
\text { o } \\
\text { ग्रा }\end{array}$ & $\begin{array}{l}\stackrel{7}{0} \\
\stackrel{D}{0}\end{array}$ \\
\hline 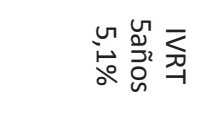 & 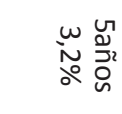 & స & 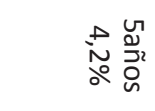 & 1 & $\sum_{\substack{j \\
-1}}$ & 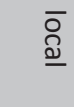 \\
\hline 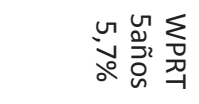 & 1 & 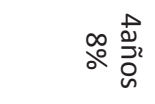 & ¿ั & 1 & $\begin{array}{l}z \\
\text { o } \\
\text { ग्] }\end{array}$ & 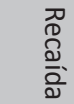 \\
\hline 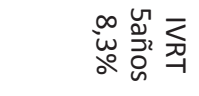 & 1 & जূ & 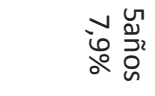 & 1 & $\sum_{\substack{0 \\
-1}}$ & 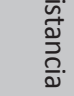 \\
\hline 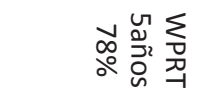 & 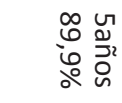 & 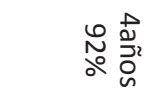 & 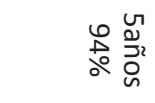 & 1 & $\begin{array}{l}z \\
\text { o } \\
\text { ग्] }\end{array}$ & \\
\hline 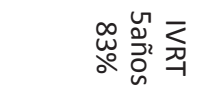 & 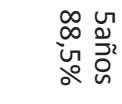 & 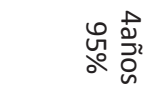 & 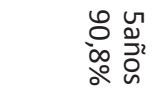 & 1 & 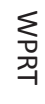 & \\
\hline 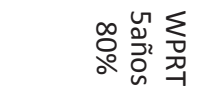 & 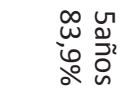 & 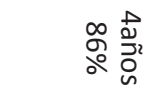 & 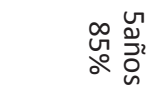 & 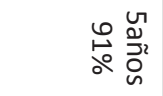 & $\begin{array}{l}z \\
\text { ग्र }\end{array}$ & \\
\hline 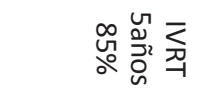 & 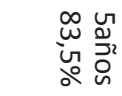 & 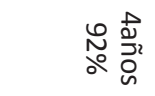 & 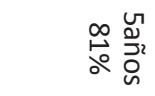 & 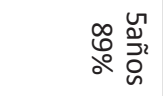 & $\sum_{\substack{0 \\
-1}}$ & \\
\hline
\end{tabular}

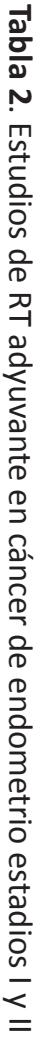




\section{CÁNCER DE OVARIO}

\subsection{El cáncer de ovario en cifras}

El cáncer de ovario es el 60 tumor por orden de frecuencia y la 5a causa de muerte por cáncer en la mujer europea. Con respecto a otros tumores ginecológicos representa el segundo más frecuente por detrás del cáncer de útero pero es el tumor ginecológico que más muertes causa. El motivo principal de esta alta mortalidad del cáncer de ovario se debe a que la mayoría de las pacientes se diagnostican en una fase avanzada de la enfermedad debido a la ausencia de técnicas de diagnóstico precoz.

Existen 3 tipos fundamentales de tumores malignos de ovario:

- Carcinoma epitelial: representa el 85-90\% de los canceres de ovario y es el que vamos a desarrollar en adelante.

- Tumores de células germinales. Muy infrecuentes.

- Tumores del estroma. Aún más infrecuentes.

En cuanto a la edad de presentación, el cáncer epitelial de ovario es una enfermedad más frecuente en las mujeres postmenopaúsicas, con la máxima incidencia entre los 50 y 75 años. Existen diferencias geográficas en la incidencia de esta enfermedad, siendo más frecuente en países industrializados.

\subsection{Aspectos biológicos del cáncer de ovario}

El cáncer epitelial de ovario no se puede considerar una única enfermedad sino al menos un conjunto formado por 5 diferentes tipos histológicos:

- Carcinoma seroso de bajo grado.

- Carcinoma endometriode.

- Carcinoma mucinoso.

- Carcinoma de células claras.

- Carcinoma seroso de alto grado.

Además de la diferente apariencia morfológica e histológica, los diferentes subtipos tienen un diferente comportamiento clínico y pronóstico. Así por ejemplo, los tumores serosos de bajo grado, mucinosos y de células claras suelen presentarse mayoritariamente en estadios iniciales (etapas I y II) mientras que los subtipos endometriode de alto grado y seroso de alto grado suelen aparecer en etapas avanzadas (estadios III y IV de FIGO). Con respecto al pronóstico, las etapas III y IV son la que presentan un peor pronóstico, y den- tro de este grupo las histología mucinosa o de células claras inducen una peor supervivencia.

Pero más allá de estas diferencias, entre los distintos subtipos subyacen también diferentes alteraciones moleculares que explican el diferente comportamiento y que además son motivo actual del desarrollo de terapias dirigidas (Bast, Hennessy y Mills, 2009). Uno de los subtipos que se encuentra mejor caracterizado es el carcinoma seroso de alto grado, que a su vez es el tipo histológico más frecuente puesto que representa el $80 \%$ de los tumores avanzados. El proyecto TCGA (The Cancer Genome Atlas) realizó un análisis genómico masivo en más de 400 tumores serosos de alto grado que ha permitido conocer con detalle las alteraciones moleculares subyacentes en este tipo de tumor (Bell et al., 2011). Cabe destacar que un 95\% de las pacientes presentan una mutación de p53 y que el 50\% aproximadamente tienen un déficit en recombinación homóloga, que es el sistema de reparación de la rotura de doble cadena de DNA. Este último aspecto tiene especial relevancia, puesto que incluye las mutaciones de los genes BRCA 1 y BRCA 2 (aproximadamente un $18 \%$ de pacientes con tumores serosos de alto grado tiene mutación de alguno de los dos genes) que son responsables del síndrome de cáncer de mama y ovario hereditario, y porque actualmente comenzamos a disponer de terapias dirigidas para estas pacientes como veremos más adelante.

\subsection{Papel de la cirugía en el tratamiento del cáncer de ovario avanzado y controversias actuales}

Como se ha comentado previamente la mayoría de las pacientes con diagnóstico de cáncer epitelial de ovario debutan con enfermedad avanzada, es decir diseminada en la cavidad abdominal y en el peritoneo. A pesar de que la enfermedad esté extendida, y a diferencia de la mayoría de enfermedades neoplásicas, se ha demostrado que realizar una cirugía citorreductora, que significa la extirpación de la mayor cantidad de enfermedad posible, tiene un impacto en la supervivencia de las pacientes. De hecho, el volumen de enfermedad residual tras la cirugía inicial es el factor pronóstico más importante en cáncer de ovario avanzado, en la medida en que la supervivencia a 5 años puede ser hasta el doble para las pacientes sin enfermedad residual, en comparación con las pacientes en las que queda enfermedad residual visible tras la cirugía (60\% vs 25-35\%) (du Bois et al., 2009a).

Por este motivo, la primera intervención terapéutica en cáncer de ovario avanzado debe ser una cirugía citorreductora con el objetivo de dejar a la paciente 
sin enfermedad residual macroscópica, lo que se denomina citorreducción óptima.

La cirugía necesaria para obtener el objetivo de citorreducción completa, es a veces compleja y requiere la realización de procedimientos específicos que entre otros incluyen una linfadenectomía retroperitoneal y pélvica, resección de segmentos de intestino, colon o recto con su correspondiente re-anastomosis, extirpación del peritoneo en varias localizaciones o incluso en su totalidad, y resección de enfermedad en localizaciones específicas como diafragma, bazo o hígado. Por ello, se recomienda que las pacientes sean intervenidas por ginecólogos con el adecuado nivel de entrenamiento o equipos multidisciplinares bien integrados. Además, se dispone de datos que indican que cuando las pacientes son intervenidas por equipos especializados obtienen una mayor tasa de citorreducción óptima y de supervivencia (du Bois et al., 2009b).

\subsection{Tratamiento con quimioterapia del cáncer de ovario}

Las pacientes con cáncer de ovario avanzado deben de recibir un tratamiento médico complementario a la cirugía, incluso en aquellas pacientes en las que se consiga una citorreducción completa u óptima. El carcinoma epitelial de ovario avanzado es una enfermedad que se considera quimiosensible en la medida en que la tasa de respuesta a quimioterapia basada en platino se sitúa por encima del $70 \%$.

El tratamiento de quimioterapia básico del cáncer epitelial de ovario avanzado consiste en una combinación de paclitaxel y carboplatino administrados por vía intravenosa cada 3 semanas. Los grandes hitos históricos que condujeron a la aceptación de este tratamiento como el estándar de quimioterapia fueron: 1) la incorporación de sales de platino en los 80 (especialmente cisplatino), 2) la incorporación de paclitaxel en combinación con cisplatino a mediados de los 90, y 3) la constatación a finales de los 90 de que la combinación de paclitaxel y carboplatino tenía la misma eficacia y menores efectos adversos que paclitaxel y cisplatino (Gonzalez-Martin et al., 2014; Stuart et al., 2011).

Este esquema ha sufrido algunas variaciones en la frecuencia de administración (dosis densas) o en la ruta de administración (quimioterapia intraperitoneal) que han sido motivo de estudio y que aún resultan controvertidos. El concepto de dosis densas hace referencia a la administración de uno de los dos fámacos (habitualmente paclitaxel) o ambos (paclitaxel y carboplatino) con una mayor periodicidad (en general semanal). Aunque un estudio realizado en población japonesa demostró un incremento tanto de la supervivencia libre de progresión como de la supervivencia global, otro estudio realizado en Italia con población caucásica no pudo demostrar este beneficio (Katsumata et al., 2012). Por ello, a la espera de un gran estudio internacional que está siendo liderado por el grupo MRC del Reino Unido, aún no se puede considerar el esquema de dosis densas como el tratamiento de elección para las pacientes. Por su parte, la quimioterapia intraperitoneal consiste en la administración de toda o, más frecuentemente, parte de los ciclos de quimioterapia directamente en la cavidad peritoneal a través de un catéter. Varios estudios han explorado esta intervención terapéutica en comparación con la administración intravenosa demostrando un impacto en la supervivencia libre de enfermedad y en algún caso en la supervivencia global con una reducción del riesgo de muerte de aproximadamente un $20 \%$ en los resultados de un meta-análisis (Armstrong, Bunden y Wenel, 2006). A pesar de esta ventaja observada en la supervivencia global, esta tecnología no se ha implementado de forma generalizada porque el tratamiento intraperitoneal se asocia a una mayor complejidad de administración, tasa de complicaciones y efectos adversos en comparación con el tratamiento intravenoso. Pero dado los resultados en supervivencia debería ser contemplada como una opción en pacientes con enfermedad avanzada y buena situación clínica en las que queda enfermedad residual mínima $(<1$ $\mathrm{cm})$ tras cirugía citorreductora.

\subsection{Avances en el tratamiento médico del cáncer de ovario}

El avance del tratamiento médico del cáncer epitelial de ovario ha venido del desarrollo de terapia biológica dirigida, especialmente de la terapia antiangiogénica y de la terapia con inhibidores de la enzima PARP que aprovechan la existencia de alteraciones en los mecanismos de recombinación homóloga implicados en la reparación del DNA.

La neoangiogénesis, o desarrollo de nuevos vasos sanguíneos, juega un papel esencial en la proliferación y progresión de la enfermedad, y se ha demostrado que es un factor pronóstico adverso en cáncer de ovario. Entre los diferentes mecanismos implicados en la angiogénesis, la interacción del factor de crecimiento vascular endotelial (VEGF) con su receptor (VEGFR) se ha considerado el más relevante. Actualmente disponemos de terapias con capacidad de bloquear tanto el ligando (VEGF) como su receptor (VEGFR). En con- 
creto, ya contamos con un agente antiangiogénico llamado bevacizumab aprobado para su uso en la clínica para el tratamiento de pacientes con cáncer de ovario tanto en la primera línea como en la recaída. Bevacizumab, es un anticuerpo monoclonal humanizado que bloquea VEGF impidiendo su unión al receptor. Actualmente disponemos de datos que muestran que asociar bevacizumab a quimioterapia seguido de un periodo de mantenimiento con bevacizumab tras la cirugía inicial aumenta el intervalo libre de progresión en pacientes con diagnóstico reciente de cáncer de ovario avanzado con una reducción del riesgo de progresión de aproximadamente el 30\%. Adicionalmente, las pacientes con estadios IV y aquellas con enfermedad residual tras la cirugía inicial mayor de $1 \mathrm{~cm}$ pueden obtener también un beneficio en supervivencia global (Burger et al., 2011; Perren et al., 2011).

Junto a la terapia antiangiogénica, la otra diana prometedora en cáncer de ovario es el déficit de recombinación homóloga. Los mecanismos de recombinación homóloga están integrados por varios sistemas cuyo objetivo es reparar la ruptura de la doble cadena de DNA que ocurren especialmente durante los procesos de división celular. Aproximadamente la mitad de las pacientes con carcinoma seroso de alto grado presentan esta alteración, y en concreto casi el $20 \%$ de las pacientes tiene una mutación de los genes BRCA1 o BRCA2. Las pacientes con esta alteración pueden ob- tener un beneficio de una nueva clase de fármacos denominados inhibidores de PARP. La enzima PARP contribuye a la reparación de la rotura de cadena simple de DNA. De manera que si se inhibe PARP, la célula acumula rupturas de cadena simple de DNA durante la replicación celular que pueden ocasionar ruptura de doble cadena. Así, aquellas células que no dispongan de función adecuada de BRCA (por ejemplo por mutación del gen) entraran en apoptosis (muerte celular) al no disponer de mecanismos de reparación de la ruptura de la doble hélice de DNA (Ashworth, 2008). Actualmente disponemos de varios fármacos con actividad inhibidora de PARP en desarrollo y uno en concreto, olaparib, ha demostrado que empleado como terapia de mantenimiento es capaz de prolongar el periodo libre de progresión en pacientes con mutación de BRCA, que tras una recaída por encima de 6 meses han respondido a tratamiento con platino (Ledermann et al., 2012).

Existen otros fármacos en desarrollo, especialmente dirigidos a subgrupos de pacientes con alteraciones moleculares específicas. Como por ejemplo, inhibidores de MEK en pacientes con carcinoma seroso de bajo grado, anticuerpo monoclonal antiHER3 (pertuzumab) en pacientes con niveles bajos de RNA mensajero de HER3, o fármacos capaces de restaurar la función de p53 en pacientes con tumores serosos de alto grado en los que casi el 95\% están mutados.

\section{BIBLIOGRAFÍA}

Aalders, J., Abeler, V., Kolstad, P. y Onsrud, M. (1980). Postoperative external irradiation and prognostic parameters in stage I endometrial carcinoma: clinical and histopathologic study of 540 patients. Obstetrics \& Gynecolgy, 56, 4, pp. 419-427.

Armstrong, D., Bunden, B. y Wenel, L. (2006). Phase III randomized trial of intravenous cisplatin and paclitaxel versus an intensive regimen of intravenous paclitaxel, intraperitoneal cisplatin, and intraperitoneal paclitaxel in stage III ovarian cancer: a Gynecologic Oncology Group study. New England Journal of Medicine, 354, pp. 34-43.

Ashworth, A. (2008). A synthetic lethal therapeutic approach: poly(ADP) ribose polymerase inhibitors for the treatment of cancers deficient in DNA double-strand break repair. Journal of Clinical Oncology, 26, 22, pp. 37853790. DOI: $10.1200 / J C O .2008 .16 .0812 J$ CO.2008.16.0812 [pii].
Bast, R. C., Jr., Hennessy, B. y Mills, G. B. (2009). The biology of ovarian cancer: new opportunities for translation. $\mathrm{Na}$ ture Reviews Cancer, 9, 6, pp. 415-428. DOI: nrc2644 [pii]10.1038/nrc2644.

Bell, D., Berchuck, A., Birrer, M., Chien, J., Cramer, D. Dao, F., Dhir, R., DiSaia, P., Gabra, H. et al. (2011). Integrated genomic analyses of ovarian carcinoma. Nature, 474, 7353, pp. 609-615. DOI: nature10166 [pii]10.1038/nature10166.

Burger, R. A., Brady, M. F., Bookman, M. A., Fleming, G. F., Monk, B. J., Huang, H. et al. (2011). Incorporation of bevacizumab in the primary treatment of ovarian cancer. New England Journal of Medicine, 365, 26, pp. 2473-2483. DOI: 10.1056/NEJMoa1104390.

Burke, W. M., Orr, J., Leitao, M., Salom, E., Gehrig, P., Olawaiye, A. B. et al. (2014a). Endometrial cancer: a review and current management strategies: part
II. Gynecologic Oncology, 134, 2, pp. 393-402. DOI: S0090-8258(14)01028-2 [pii]10.1016/j.ygyno.2014.06.003.

Burke, W. M., Orr, J., Leitao, M., Salom, E., Gehrig, P., Olawaiye, A. B. et al. (2014b). Endometrial cancer: a review and current management strategies: part I. Gynecologic Oncology, 134, 2, pp. 385-392. DOI: S0090-8258(14)00978-0 [pii]10.1016/j.ygyno.2014.05.018.

Creutzberg, C. L., van Putten, W. L., Koper, P. C., Lybeert, M. L., Jobsen, J. J., Warlam-Rodenhuis, C. C. et al. (2000). Surgery and postoperative radiotherapy versus surgery alone for patients with stage-1 endometrial carcinoma: multicentre randomised trial. PORTEC Study Group. Post Operative Radiation Therapy in Endometrial Carcinoma. Lancet, 355, 9213, pp. 1404-1411. DOI: S0140673600021395 [pii].

du Bois, A., Reuss, A., Pujade-Lauraine, E., Harter, P., Ray-Coquard, I. y Pfisterer, 
J. (2009a). Role of surgical outcome as prognostic factor in advanced epithelial ovarian cancer: a combined exploratory analysis of 3 prospectively randomized phase 3 multicenter trials: by the Arbeitsgemeinschaft Gynaekologische Onkologie Studiengruppe Ovarialkarzinom (AGO-OVAR) and the Groupe d'Investigateurs Nationaux Pour les Etudes des Cancers de l'Ovaire (GINECO). Cancer, 115, 6, pp. 1234-1244. DOI: 10.1002/cncr.24149.

du Bois, A., Rochon, J., Pfisterer, J. y Hoskins, W. J. (2009b). Variations in institutional infrastructure, physician specialization and experience, and outcome in ovarian cancer: a systematic review. Gynecologic Oncology, 112, 2, pp. 422-436. DOI: S0090-8258(08)00810-X [pii]10.1016/j.ygyno.2008.09.036.

Gonzalez-Martin, A., Bover, I., Del Campo, J. M., Redondo, A. y Vidal, L. (2014). SEOM guideline in ovarian cancer 2014. Clinical and Translational Oncology, 16, 12, pp. 1067-1071. DOI: 10.1007/ s12094-014-1229-z.

Hogberg, T., Signorelli, M., de Oliveira, C. F., Fossati, R., Lissoni, A. A., Sorbe, B. et al. (2010). Sequential adjuvant chemotherapy and radiotherapy in endometrial cancer--results from two randomised studies. European Journal of Cancer, 46, 13, pp. 2422-2431. DOI: S0959-8049(10)00477-6 [pii]10.1016/j. ejca.2010.06.002.

Katsumata, N., Yasuda, M., Isonishi, S., Michimae, H., Kimura, E., Aoki, D. et al. (2012). Long-term follow-up of a randomized trial comparing conventional paclitaxel and carboplatin with dosedense weekly paclitaxel and carboplatin in women with advanced epithelial ovarian, fallopian tube, or primary peritoneal cancer: JGOG 3016 trial. ASCO Meeting Abstracts, 30, abstract 5003.

Keys, H. M., Roberts, J. A., Brunetto, V. L., Zaino, R. J., Spirtos, N. M., Bloss, J. D. et al. (2004). A phase III trial of surgery with or without adjunctive external pelvic radiation therapy in intermediate risk endometrial adenocarcinoma: a Gynecologic Oncology Group study. Gynecologic Oncology, 92, 3, pp. 744751. DOI: $10.1016 /$ j.ygyno.2003.11.04 850090825803008631 [pii].

Kitchener, H., Swart, A. M., Qian, Q., Amos, C. y Parmar, M. K. (2009). Efficacy of systematic pelvic lymphadenectomy in endometrial cancer (MRC ASTEC trial): a randomised study. Lancet, 373, 9658, pp. 125-136. DOI: S01406736(08)61766-3 [pii]10.1016/S01406736(08)61766-3.

Ledermann, J., Harter, P., Gourley, C., Friedlander, M., Vergote, I., Rustin, G. et al. (2012). Olaparib maintenance therapy in platinum-sensitive relapsed ovarian cancer. New England Journal of Medicine, 366, 15, pp. 1382-1392. DOI: 10.1056/NEJMoa1105535.

McGraw, S. L. y Ferrante, J. M. (2014). Update on prevention and screening of cervical cancer. World Journal of Clinical Oncology, 5, 4, pp. 744-752. DOI: 10.5306/wjco.v5.i4.744.

Nout, R. A., Smit, V. T., Putter, H., Jurgenliemk-Schulz, I. M., Jobsen, J. J., Lutgens, L. C. et al. (2010). Vaginal brachytherapy versus pelvic external beam radiotherapy for patients with endometrial cancer of high-intermediate risk (PORTEC-2): an open-label, non-inferiority, randomised trial. Lancet, 375, 9717, pp. 816-823. DOI: S0140-6736(09)62163-2 [pii]10.1016/S0140-6736(09)62163-2.

Perren, T. J., Swart, A. M., Pfisterer, J., Ledermann, J. A., Pujade-Lauraine, E., Kristensen, G. et al. (2011). A phase 3 trial of bevacizumab in ovarian cancer. New England Journal of Medicine, 365, 26, pp. 2484-2496. DOI: 10.1056/NEJMoa1103799.

Shih, K. K., Yun, E., Gardner, G. J., Barakat, R. R., Chi, D. S. y Leitao, M. M., Jr.
(2011). Surgical cytoreduction in stage IV endometrioid endometrial carcinoma. Gynecologic Oncology, 122, 3, pp. 608-611. DOI: S0090-8258(11)00417-3 [pii]10.1016/j.ygyno.2011.05.020.

Stuart, G. C., Kitchener, H., Bacon, M., duBois, A., Friedlander, M., Ledermann, J. et al. (2011). 2010 Gynecologic Cancer InterGroup (GCIG) consensus statement on clinical trials in ovarian cancer: report from the Fourth Ovarian Cancer Consensus Conference. International Journal of Gynecological Cancer, 21, 4, pp. 750-755. DOI: 10.1097/IGC.0b013e318 21b256800009577-201105000-00027 [pii].

Susumu, N., Sagae, S., Udagawa, Y., Niwa, K., Kuramoto, H., Satoh, S. et al. (2008). Randomized phase III trial of pelvic radiotherapy versus cisplatin-based combined chemotherapy in patients with intermediate- and high-risk endometrial cancer: a Japanese Gynecologic Oncology Group study. Gynecologic Oncology, 108, 1, pp. 226-233. DOI: S0090-8258(07)00786-X [pii]10.1016/j. ygyno.2007.09.029.

Tewari, K. S. y Monk, B. J. (2014). New strategies in advanced cervical cancer: from angiogenesis blockade to immunotherapy. Clinical Cancer Research, 20, 21, pp. 5349-5358. DOI: 1078-0432. CCR-14-1099 [pii]10.1158/1078-0432. CCR-14-1099.

Tewari, K. S., Sill, M. W., Long, H. J., 3rd, Penson, R. T., Huang, H., Ramondetta, L. M. et al. (2014). Improved survival with bevacizumab in advanced cervical cancer. New England Journal of Medicine, 370, 8, pp. 734-743. DOI: 10.1056/NEJMoa1309748.

Wright, J. D., Barrena Medel, N. I., Sehouli, J., Fujiwara, K. y Herzog, T. J. (2012). Contemporary management of endometrial cancer. Lancet, 379, 9823, pp. 1352-1360. DOI: S0140-6736(12)604425 [pii]10.1016/S0140-6736(12)60442-5. 